\title{
DESIGNING NANOSTRUCTURES FROM POLY (STYRENE-B-BUTADIENE-B-METHYL METHACRYLATE) (SBM) TRIBLOCK COPOLYMERS
}

\author{
L. $\operatorname{Martin}^{1}$, A. Tercjak ${ }^{1}$, G. Kortaberria ${ }^{1 *}$ \\ 1 "Materials + Technologies" Group, Chemical Engineering Department, Escuela de Ingeniería de Gipuzkoa, \\ Spain
}

\begin{abstract}
New and more complex morphologies can be obtained by preparing blends of SBM triblock copolymers with different block amounts or blending triblock copolymers with diblock SB ones with the same block ratio. Thin films of those blends have been prepared by spin coating and morphologies analyzed by atomic force microscopy (AFM). Complex morphologies such as asymmetric lamellae, cylinders at cylinders (cac), cylinders in cylinders (cic) or knitting pattern, predicted by the ternary diagram, have been obtained and described in terms of block amount or interactions among them.
\end{abstract}

Keywords: block copolymers, nanostructure, atomic force microscopy Introduction

\section{Introduction}

The spontaneous formation of nanostructured materials by molecular self-assembly of block copolymers is an active area of research, driven mainly by a wealth of potential technological applications (Barandiaran and Kortaberria, 2013, Barandiaran et al, 2015). Thin films of block copolymers have attracted increasing interest, particularly in view of possible applications in nanotechnology (Lazzari and Lopez-Quintela, 2003, Huang et al, 2012, Gotrik et al, 2012). Although much of the work has concentrated on block copolymers consisting of two components, the insertion of a third block greatly enlarges the structural diversity and allows incorporation of additional chemical functionality. Among triblock copolymers, ABC-type ones can be underlined. Morphologies generated for ABC-type copolymer thin films have been investigated by several authors, who analyzed the effect of substrates, solvents and interactions on obtained morphologies. In this way, Fukunaga et al (2003) analyzed the effect of substrate on morphology, Elbs et al (2002) analyzed the effect of solvent vapors on morphology of SVT copolymer thin films and Bercea et al (2017) studied the effect of selective solvent and temperature on the morphology of PSU-b-PAO-b-PDMS copolymers.

Regarding SBM-type block copolymers, the work of Abetz et al must be mentioned (2000). They analyzed both experimentally and theoretically the effect of block ratio on the morphology of thin films, preparing the corresponding ternary diagram. Following this diagram,, our group has recently prepared thin films of different SBM block copolymers prepared with different solvents (L. Martin et al, 2017a) or thin films of mixtures of SBM copolymers with PS and/or PMMA homopolymers (L.Martin et al, 2017b) obtaining complex morphologies predicted by the ternary diagram proposed by Abetz et al, such as cylinders on cylinders (coc), perforated lamellae, spheres on spheres (sos), among others. 
In the present work, thin films of blends of different SBM copolymers with SB diblock copolymers and blends of different SBM copolymers have been prepared and their morphology has been analyzed by atomic force microscopy (AFM).

Experimental

\section{Materials}

Two SBM copolymers with different block ratios and molar masses, synthesized by anionic polymerization at pilot scale by Arkema, were used: SBM-1 (96000 g/mol and 31/36/33 block weigth ratio) and SBM-2 (88000 $\mathrm{g} / \mathrm{mol}$ and 40/06/54 ratio). Chloroform, used as solvent for thin film preparation, was purchased from SigmaAldrich.

\section{Characterization techniques}

Morphological characterization of thin films was carried out by AFM, with a scanning probe atomic force microscope with a Nanoscope IIIa controller (Veeco) and a Dimension ICON atomic force microscope with a Nanoscope V controller (Bruker), both operating in tapping mode. An integrated silicon tip/cantilever with a resonance frequency of around $300 \mathrm{kHz}$ was used, performing measurements at a scan rate of $1 \mathrm{~Hz} \mathrm{~s}^{-1}$ with 512 scan lines. Different areas of each sample were analysed to ensure the final morphology of the investigated blends.

\section{Sample preparation}

SBM triblock/diblock or triblock/triblock blend thin films were prepared by spin-coating onto $\operatorname{Si}(100)$ wafers at $2000 \mathrm{rpm}$ for $120 \mathrm{~s}$ using a Specialty Coating Systems P-6708D spin-coater. In order to prepare the polymer blend films, a $10 \%$ solution of each polymer in chloroform was spin-coated. The thickness of the films was around $300 \mathrm{~nm}$ as measured by AFM.

\section{Results and discussion}

\section{SBM/SB blends}

Two different triblock/diblock copolymer blends, in which both present the same block ratio were prepared and analyzed: SBM-1/SB (53/47), and SBM-2/SB (59/41). The first blend, with a volumetric composition of $36 / 49 / 15$, would correspond to a lamellar morphology if we take into account its position in the ternary diagram. As it can be seen in the AFM image of Figure 1, a lamellar super-structure was obtained, but the symmetry of lamellae was completely desestabilized when compared with pure copolymer (Martin et al, 2017a). It has been pointed out by some authors that, with the addition of shorter chains of the diblock copolymer, the change in the curvature of long chains corresponding to the triblock copolymer could change the curvature of lamellae, with the obtention of non-centrosymmetric morphologies that cannot be obtained for pure ABC copolymers (Goldacker et al, 1999).

The AFM image corresponding to SBM-2/SB blend, with a volumetric composition of 60/11/29 can be seen in Figure 2. When placed at the ternary diagram, it lies at the transition zone between $1_{\mathrm{s}}$ morphology (consisting on PB spheres at PS/PMMA interface) obtained for pure SBM-2 with the same solvent (Martin et al, 2017a) and cylinders at cylinders $\left(c_{a} c\right)$ one. As it can be seen in the image, PB cylinders appear surrounding PMMA ones, in a PS matrix. The morphology is very close to $c_{a} c$ one obtained for the blend of SBM-1 with PS and PMMA homopolymers (Martin et al, 2017b). The difference is that for SBM-2/SB blend, PB shells are continuous and donot appear cut or discontinuous like they were for SBM-1/PS/PMMA blend, the morphology seeming to be closer to cylinders in cylinders $\left(\mathrm{c}_{\mathrm{i}} \mathrm{c}\right)$ one. 


\section{SBM/SBM blend}

Blends of SBM copolymers could lead to complex architectures. In this way, Bretner et al (1998), prepared blends of SEBM copolymers obtaining a knitting pattern morphology, described as an intermediate morphology between lamellar and EB cylinders at S/M interface. In this morphology, M forms peristaltic lamellae with a big gap among maxims and minims of consecutive lamellae. Cylindrical domains of EB with elliptical shape are situated at those valleys. Schematic representation can be seen in Figure 3, together with the image corresponding to SBM-1/SBM-2. With the aim of obtaining a similar morphology and taking into account the position at the ternary diagram, SBM-1/SBM-2 (50/50) blend was prepared. SBM-2 presented a lc morphology (B cylinders at $\mathrm{S} / \mathrm{M}$ interface) (Martin et al, 2017a) in accordance with the volumetric fraction of PB block (0.08). The 50/50 blend (with an increased volume fraction of 0.25 for PB block), would be placed in the ternary diagram in an intermediate position between lc and lamellar morphologies. As it can be seen in Figure 3, M domains present a continuous wavy form as pointed out by Breiner, but much more curved. B domains donot present any elliptical form. Those discrepancies could be due to differences in interaction energies corresponding to SB and BM pairs when compared with SEB and EBM ones.

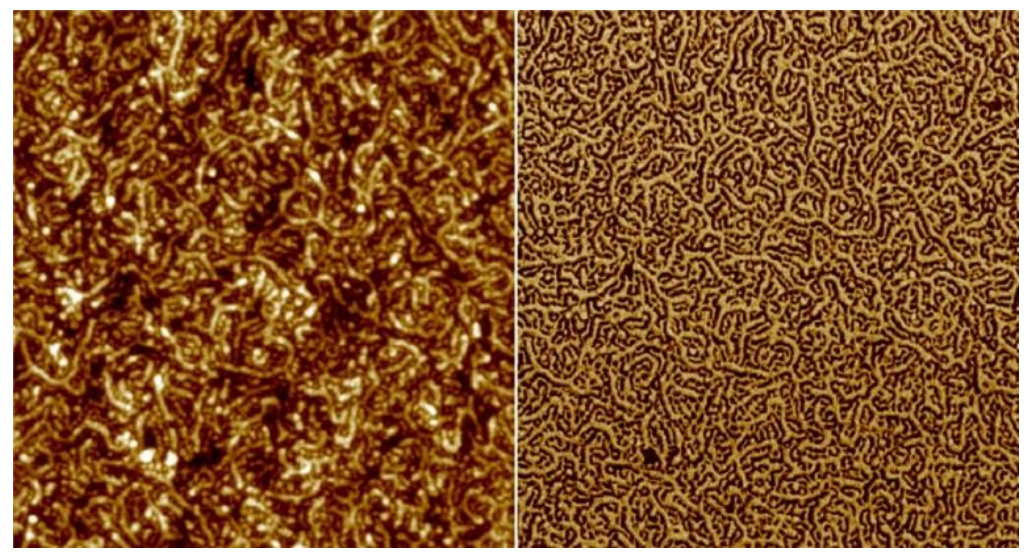

Figure 1 Heigth (left) and phase (right) AFM images of a $3 \mu \mathrm{m} \times 3 \mu \mathrm{m}$ thin film of SBM-1/SB blend spin coated from a chloroform solution 


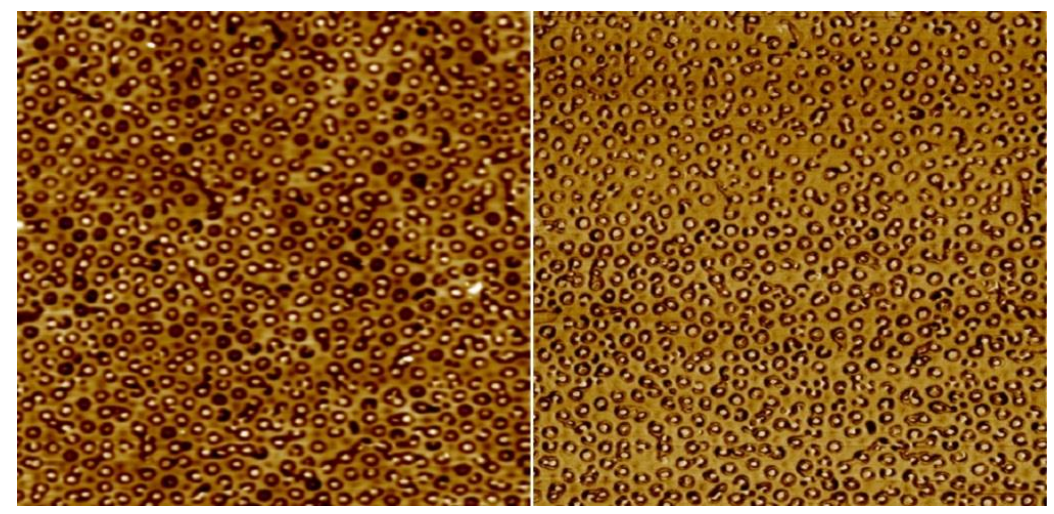

Figure 2 Heigth (left) and phase (right) AFM images of a $3 \mu \mathrm{m} \times 3 \mu \mathrm{m}$ thin film of SBM-2/SB blend spin coated from a chloroform solution


Figure 3 Heigth (left) and phase (right) AFM images of a $3 \mu \mathrm{m} \times 3$ um thin film of SBM-1/SBM-2 blend spin coated from a chloroform solution, together with a schematic representation of the knitting pattern morphology obtained by Breiner et al

\section{Conclusions}

Taking into account the ternary diagram and preparing blends of SBM copolymers or blends with SB diblock copolymers adjusting block ratio, complex morphologies such as non-centrosymmetric, cylinders in cylinders or knitting pattern can be predicted and obtained in an easy and simple way.

\section{Acknowledgements}

Financial support from the Basque Country Government (Grupos Consolidados IT776-13), and the Ministry of Economy and Competitiveness (MAT2015) is gratefully acknowledged. Technical and human support provided by SGIker is also acknowledged.

\section{References}

ABETZ, V., GOLDACKER, T., 2000, Formation of superlattices via blending of block copolymers. Macromolecular Rapid Communications, 21, 16-34. 
BARANDIARAN, I., KATSIGIANNOPOUlOS, D., GRANA, E., AVGEROPOUlOS, A., ECEIZA, A., KORTABERRIA, G., 2013, PI-b-PMMA diblock copolymers: nanostructure development in thin films and nanostructuring of thermosetting epoxy systems. Colloid and Polymer Science, 291, 2173-2180.

BARANDIARAN, I., KORTABERRIA, G., 2015, Synthesis and characterization of nanostructured PS-b$\mathrm{PVP} / \mathrm{Fe}_{2} \mathrm{O}_{3}$ thin films with magnetic properties by solvent vapor annealing. RSC Advances, 5, 95840-95846.

BERCEA, M., AIRINEI, A., HAMCIUC, V., 2017, Temperature influence on the behavior of polysulfone-bpoly(alkylene oxide)-b-poly(dimethylsiloxane) triblock copolymers in a selective solvent. Polymer Engineering and Science, 57, 114-122

BREINER, U., KRAPPE, U., THOMAS, E. L., STADLER, R., 1998. Structural characterization of the knitting pattern in polystyrene-b-(ethylene-co-butylene)-b-(methyl methacrylate) triblock copolymers. Macromolecules, 31, 135-141.

ELBS, H., DRUMMER, C., ABETZ, V., KRAUSCH, G., 2002, Thin film morphologies of ABC triblock copolymers prepared from solution. Macromolecules, 35, 5570-5577.

FUKUNAGA, T., HASHIMOTO, T., ELBS, H., KRAUSCH, G., 2003, Self-assembly of a lamellar ABC triblock copolymer thin film. Effect of substrates. Macromolecules, 36, 2852-2861.

GOLDACKER, T., ABETZ, V., 1999. Non-centrosymmetric superlattices in block copolymer blends. Letters to Nature, 398, 137-139.

GOTRIK, K. W., HANNON, J. G., KELLER, B., ALEXANDER-KATZ, A., ROSS, C. A., 2012, Morphology control in block copolymer films using mixed solvent vapors. ACS Nano, 6, 8052-8059.

HUANG, W. H., CHEN, P. Y., TUNG, S. H., 2012, Effects of annealing solvents on the morphology of block copolymer-based supramolecular thin films. Macromolecules, 45, 1562-1569.

LAZZARI, M., LOPEZ-QUINTELA, M. A., 2003, Block copolymer as a tool for nanomaterial fabrication. Advanced Materials, 15, 1583-1594.

MARTIN, L., IRUSTA, L., GONZALEZ, A., TERCJAK, A., KORTABERRIA, G., 2017. Nanostructure development in polystyrene-b-polybutadiene-b-poly(methyl methacrylate) (SBM) thin films by atomic force microscopy: Effect of copolymer composition and solvent. Polymer Engineering and Science, DOI: $10.1002 /$ pen.24589.

MARTIN, L., IRUSTA, L., GONZALEZ, A., TERCJAK, A., KORTABERRIA, G., 2017. Nanostructured polymer blends based on polystyrene-b-polybutadiene-b-poly(methyl methacrylate) triblock copolymers modified with polystyrene and/or poly(methyl methacrylate) homopolymers. Polymer International, 66, 10 